\title{
Physical Cosmic Strings Do Not Generate Closed Timelike Curves
}

\author{
S. Deser \\ Physics Department, Brandeis University, Waltham, Massachusetts 02254 \\ R. Jackiw \\ Center for Theoretical Physics, Laboratory for Nuclear Science and Department of Physics, \\ Massachusetts Institute of Technology, Cambridge, Massachusetts 02139 \\ G. 't Hooft \\ Institute for Theoretical Physics, Princetonplein 5, P.O. Box 80006, 3508 TA Utrecht, The Netherlands \\ (Received 21 August 1991)

\begin{abstract}
We reexamine the causal properties of geometries generated by parallel, moving cosmic strings, particularly our statement that closed timelike curves are forbidden there. Contrary to a recent claim, such acausal behavior cannot be realized by physical, timelike, sources.
\end{abstract} \\ PACS numbers: $04.20 . J b, 04.20 . \mathrm{Cv}, 98.80 . \mathrm{Cq}$
}

Some time ago [1], we discussed the global properties of the (locally flat) geometries generated by moving point particles in $2+1$ dimensions, or equivalently by parallel moving cosmic strings in $3+1$ dimensions. Let us call sources of either type "cosmons" for brevity. We stated without proof that closed timelike curves (CTC's) are forbidden in universes generated by spinless cosmons, although they could be present in generic exterior "Kerr" geometries. Physically, our reason was that the cosmon system, like any conventional general relativistic one, should be constructable by Cauchy evolution of spacelike surfaces, and we saw no ground for spontaneous formation of obstructions (such as CTC's) in the evolution of normal cosmons. We show explicitly here, using the solutions in [1], that our conclusion is indeed correct: There are no CTC's if the space-times have physically acceptable global structure, which they do for physically acceptable sources.

A recent interesting construction [2] of a space-time generated by two moving cosmons and supporting CTC is not in contradiction with our statement, because that construction does not obey our "physical acceptability" criteria (which were not spelled out in Ref. [1]), viz., there must not be CTC at spacelike infinity or at an initial configuration. The fact that no CTC's will then arise in physically acceptable universes may seem tautological but it is not, because replacing the two cosmons carrying nonvanishing relative angular momentum by a single spinning source with the same external geometry would give rise to CTC's in its external space-time. CTC's do not arise spontaneously from regular initial conditions through the motion of spinless cosmons, whatever their velocities and resultant orbital angular momentum may be.

For orientation, we begin with the explicit metric representation of the general exterior space-time corresponding to the two conserved quantities $(M, J)$, namely, the
Kerr solution whose interval is [1]

$d s^{2}=[d(t+J \theta)]^{2}-\left[d r^{2}+r^{2} d \theta^{2}\right], \quad 0 \leq \theta \leq 2 \pi(1-M)$.

(Notation is as in [1], except that we set the gravitational coupling strength $G=\frac{1}{4}$.) The metric is manifestly locally flat outside the origin, where the Einstein tensor density has a singularity describing a massive, spinning point source: $T^{00} \propto M \delta^{2}(\mathbf{r}), T^{0 i} \propto J \epsilon^{i j} \partial_{j} \delta^{2}(\mathbf{r})$. For generic values of $(M, J)$ there are clearly CTC's in (1), namely, circles of constant $\left(t, r_{0}(t)\right)$ such that $J>r_{0}(t)$. The physical question, however, is whether moving structureless cosmon sources with purely orbital angular momentum loutside which the metric has the form (1) at any instant] can be confined within a small enough region to satisfy the above CTC criterion. If this is possible at some finite $t_{0}$, then at sufficiently large times the (freely moving) cosmons will have evolved so far apart that the CTC's would disappear spontaneously. By expressing the exterior $(M, J)$ parameters in terms of the cosmon masses and velocities, the criterion for the occurrence of CTC's will be found and shown to require unphysical sources.

The required relations between the external parameters and the constituent ones are already given in the geometrical description of space-time in Sec. $V$ of [1]. There we showed that the global properties of the exterior flat space are characterized by the identification of points according to

$$
x^{\prime}=\Omega x+c, \quad \Omega \equiv L_{\mathbf{v}} \Omega_{m} L_{\mathrm{v}}^{-1} L_{\mathrm{v}}^{-1} \Omega_{m} L_{\mathrm{v}} .
$$

Here $L_{v}$ is a Lorentz boost with velocity $\mathbf{v}$ and $\Omega_{m}$ is a spatial rotation through angle $2 \pi m$. For simplicity, we take two equal cosmon masses $m$ in the frame where their velocities are equal and opposite, $\pm \mathbf{v}$; our results are of course equally valid in any frame, as well as for more than two cosmons and different mass values. The 
geometry of the system will correspond to a single effective physical source if $\Omega$ is itself spacelike, namely, a boosted rotation, $\Omega=\Lambda \Omega_{M} \Lambda^{-1}$, where $\Omega_{M}$ is a pure rotation through $2 \pi M$ and $\Lambda$ is a boost. In the frame where $\boldsymbol{\Omega}=\boldsymbol{\Omega}_{M}$, the time component $c^{0}$ of the shift in (2) characterizes the jump in time that always occurs when angular momentum is enclosed [1].

From the fact that $\Omega$ is rotationlike, it follows that $(\operatorname{tr} \Omega-1) / 2=\cos 2 \pi M$ lies in the interval $[-1,1]$. When $\operatorname{tr} \Omega$ is expressed from (2) in terms of constituent parameters, this implies that [see (5.12) and (5.17) in [1]]

$$
\cosh \xi \sin \pi m<1,
$$

where $v=\tanh \xi$. On the other hand, Gott's construction [2] of CTC's in the two-cosmon system requires the opposite, i.e., that the left-hand side in (3) must be greater than 1 so that the composite $M$ is imaginary, and the identification in (2) is boostlike. In the most general external space-time the analog of $\Omega$ in (2) can be any Lorentz transformation; it could be equivalent to a boost rather than to a rotation, in which case CTC's are always present.

What are the properties of such systems, corresponding to a boostlike identification? Here the conical structure of the $(x, y)$ plane is replaced by one in the $(t, v)$ hyperplane, along with a jump in the remaining spatial direction rather than in time. In terms of the line element, it corresponds to a space-time rotated form of (1),

$$
\begin{gathered}
d \tau^{2}=d t^{2}-d x^{2}-\alpha \frac{(x d t-t d x)^{2}}{t^{2}-x^{2}} \\
-\left[d\left(y+\beta \ln \frac{t+x}{t-x}\right)\right]^{2}
\end{gathered}
$$

reminiscent of Misner spaces [3]. (We have taken $\mathbf{v}$ along the $x$ axis.) Note that the coefficient of the "deficit" $\alpha$ is now singular on the whole $x \pm t=0$ surface rather than just at the position of the particle, as in (1). Correspondingly, in the effective stress tensor, $T^{00} \propto \delta(\mathbf{r})$ associated with (1) becomes replaced by $T^{y y} \propto \delta(x) \delta(t)$ - a tachyonic particle. Such a "boost-identified" spacetime will never arise by boosting a "rotation-identified" space-time.

Consequently, Gott's CTC's cannot be created or destroyed, but come towards the interaction region from spacelike infinity. We can view them as resulting from a boundary condition at spacelike infinity that one should call "unphysical," namely, the identification (4). The sources must always be moving at their high velocity, in order to ensure his CTC criterion, i.e., the opposite of (3).

We emphasize that while each of Gott's two cosmons is separately subluminal, together they give rise to a tachyonic center of mass, which cannot arise in a system with physical, timelike center-of-mass momentum. This is a consequence of the nonlinear energy addition formula in multiconical space-time. It is analogous to what is found for static particles: A single particle can have mass $\leq 1$, but a nonsingular space-time cannot be realized for two particles each with acceptable mass lying between $\frac{1}{2}$ and 1 . This is a consequence of global requirements, not apparent locally.

Gott's derivation implicitly assumed the cosmons to have masses whose sum did not exceed 1. In the stationary case, the sum may be greater than 1 , but then there are necessarily present further sources such that the total is 2 , corresponding to a closed $S(2)$ space [1]. In that case, there are no CTC's supported by angular momentum either, because the angular momentum vanishes (by Gauss's law, just like total charge) and no time jumps are available. Equivalently in this case, a hypothetical CTC-which must necessarily surround cosmons with mass exceeding 1-will also surround (in the opposite sense) cosmons in the remaining set, which have $<1$ and so cannot support a CTC.

Finally, one may ask whether and how the spacelike part of Gott's and similar universes can be closed so that the topology becomes $S(2) \times R(1)$ instead of $R(3)$; as noted above this is always possible in the regular case, i.e., small and slow masses. In general, to close a $(2+1)$-dimensional universe one can either use exclusively spinless sources or introduce spinning ones as well. (If a spinning source is replaced by two or more particles with orbital angular momentum one of course recovers the first option.) But for a "fundamental" spinning source, we specify matching conditions only when a closed curve is followed around the source; these matchings are defined by a deficit angle and a time shift. More precisely, we have a space with $R(3) / R(1)$ topology (a three-space with a linelike obstruction on the source's world line) and a prescription for identifying points. Now in the case of spin it turns out that the obstruction can be moved to other locations without local changes: The space-time can be analytically extended. Indeed, the obstruction can be removed entirely without leaving any singularities (contrary to the spinless case where the conical singularity is not removable), but then the space-time acquires $R(2) \times S(1)$ topology, that is, it becomes periodic in time. Needless to say, CTC's will then be present everywhere; if one wants to avoid this one must keep the obstruction, but its precise location is arbitrary. Next consider space-times whose total energy exceeds 1 but is not tachyonic. If the total angular momentum is nonvanishing and if one chooses not to insert the obstruction corresponding to the spinning source that closes the universe, then of course one obtains CTC's. In Gott's case the situation is even worse. The closing source would have to be tachyonic. One could try to associate it with an obstruction such that there are no CTC's. But in any case, it belongs to the class of particlelike sources (another, less harmful, example being negative mass sources) that one should exclude from the initial conditions. 
This material was presented by one of us (R.J.) at the Sixth Marcel Grossmann Meeting [4]; informative discussions with conference participants, especially G. Gibbons and $\mathrm{S}$. Hawking, are gratefully acknowledged. This work is supported in part by funds provided by the $\mathrm{Na}$ tional Science Foundation under Grant No. PHY-8804561 (S.D.), and the U.S. Department of Energy (DOE) under Contract No. DE-AC02-76ER03069 (R.J.).

Note added.-After this paper was submitted, we received an article [5] in which it is shown that in Gott's universe there are spacelike surfaces both at early times and at late times where no CTC's occur. The region where CTC's do occur approaches the interaction region from infinity with the speed of light. Just because of the twisted geometry this speed of light is unusually fast here. There is no disagreement with our statement that the boundary condition at spacelike infinity (where CTC's definitely occur) should be considered unphysical. Our considerations are not a criticism of the surprising findings in Refs. [2,5]. They forced us to formulate more carefully the role played by physical requirements on the boundary conditions, both at spacelike and at timelike infinity, that are necessary to avoid CTC's.

[1] S. Deser, R. Jackiw, and G. 't Hooft, Ann. Phys. (N.Y.) 152, 220 (1984).

[2] J. R. Gott, Phys. Rev. Lett. 66, 1126 (1991); see also Time 137, 74 (1991).

[3] C. W. Misner, in Relativity Theory and Astrophysics I: Relativity and Cosmology, edited by J. Ehlers (American Mathematical Society, Providence, 1967); see also S. W. Hawking and G. F. R. Ellis, The Large Scale Structure of Space-Time (Cambridge Univ. Press, Cambridge, England, 1973).

[4] Lectures by S. W. Hawking and by R. Jackiw, in "Proceedings of the Sixth Marcel Grossmann Meeting" (World Scientific, Singapore, to be published). Aspects of CTC's in four-dimensional space-time were discussed by F. Tipler, Phys. Rev. Lett. 37, 979 (1976); Ann. Phys. (N.Y.) 108, 1 (1977).

[5] C. Cutler, Phys. Rev. D 45, 487 (1992). 